\title{
Evaluating the Relevance of Quality to Institutional Performance Assessment in Higher Education
}

\author{
JAMES S. POUNDER \\ Lingnan College, Hong Kong
}

This article describes a study which evaluated the relevance of quality to institutional performance assessment in higher education. A set of scales suitable for the valid and reliable (i.e. precise) self-rating of the effectiveness of higher education institutions in Hong Kong was developed. The method of scale development served to test the applicability of the nine effectiveness dimensions contained in the Competing Values Model of Organizational Effectiveness (Quinn and Rohrbaugh, 198I, 1983) to institutional performance assessment in Hong Kong higher education. One of the nine effectiveness dimensions is quality. Application of the scale development method failed to produce a scale capable of the valid and reliable self-rating of the quality dimension. The study highlighted the multidimensional and imprecise nature of the quality concept. It is argued that additional concepts, such as those proposed in the study, are necessary to inject precision into institutional performance assessment.

\section{Introduction}

A major development in higher education worldwide over the past two decades has been the preoccupation with institutional performance measurement. Institutions of higher education have increasingly come under governmental and societal pressure to demonstrate value for money performance (Goedegebuure et al., 1990; Liaison Committee of Rectors' Conferences, 1993; Lucier, 1992; Pounder, 1997; Segers et al., 1990; Sizer et al., 1992). One outcome of this pressure has been an attempt by higher education to apply industrial concepts, formulae and techniques to the management of higher learning establishments. Following the trend in industry, higher education has chosen to base its performance measurement initiatives on the notion of quality. Quality, however, is a notoriously ambiguous term. In the commercial world, where the notion should be at home, it defies generally agreed definition. Garvin (1988), writing in the 
management area, highlights the ambiguity inherent in the quality concept as follows:

... its synonyms range from luxury and merit to excellence and value. Different companies also appear to mean different things when they use the word as do different groups within the same firm. Without further refinement, continued ambiguity and confusion are inevitable. (Garvin, 1988: 39)

The following attempts to define quality exemplify Garvin's point: (1) 'Quality of a product depends on how well it fits patterns of consumer preferences' (Kuehn and Day, 1962: 101); (2) 'Quality is the degree to which a specific product conforms to a design or specification' (Gilmore, 1974: 16); (3) 'Quality is the degree of excellence at an acceptable price and the control of variability at an acceptable cost' (Broh, 1982: 3); (4) 'Even though quality cannot be defined you know what it is' (Pirsig, 1974: 213).

In the commercial world, it seems that quality means different things to different people. Similarly, a number of writers on higher education have acknowledged the indeterminate nature of the quality concept. Vroeijenstijn (1992), for example, has pointed to the complex, subjective nature of quality. Staropoli (1992) has noted that, in the educational context, the notion is open to several interpretations depending on the perspective of the interested parties. Bauer (1992) has indicated that the idea of quality is likely to vary with different political cultures, national traditions and education systems. Williams (1990) has termed quality in higher education 'intangible and unquantifiable'. The Liaison Committee of Rectors' Conferences (1993) has asserted that quality is a notion which cannot be grasped easily in higher education. The ambiguous nature of quality is emphasized by Reeves and Bednar (1994) who have traced the evolution of quality definitions and conclude that 'the search for a universal definition of quality and a statement of lawlike relationships has been unsuccessful' (p. 441).

This article describes a Hong Kong study which tested the relevance of quality to institutional performance assessment in higher education. The study developed a set of organizational effectiveness self-rating scales for Hong Kong higher education institutions. The scales were tested for their ability to produce valid and reliable ratings. In the course of developing the scales, the relevance of quality to institutional performance assessment in Hong Kong higher education was examined. The scales were based on the nine effectiveness dimensions contained in the Competing Values Model of Organizational Effectiveness (Quinn and Rohrbaugh, 1981, 1983) which was selected for a number of reasons. First, its authors have claimed general paradigmatic status for the model. Second, that status has been supported by the high incidence of employment of this model, or its derivatives, in management and organizational literature (Cameron and Freeman, 1989; DiPadova and Faerman, 1993; Dunk and Lysons, 1997; Edwards, 1986; Giek and Lees, 1993; Hart and Quinn, 1993; Hooijberg and Petrock, 1993; McGraw, 1993; Quinn et al., 1991; Quinn and Spreitzer, 1991; Rogers and Hildebrandt, 1993; Sendelbach, 1993; Stevens, 1996; Waller et al., 1995; Yeung et al., 1991; Zammuto and Krakower, 1989; Zammuto and O'Connor, 1992). Third, quality is one of the model's nine effectiveness dimensions. 


\section{Evaluation 6(1)}

Following the general trend, Hong Kong has quality at the centre of institutional performance measurement in higher education. Hong Kong higher education institutions are mandated to demonstrate their effectiveness by a system which emphasizes 'quality' performance through self-assessment and peer review (Pounder, 1997). The study was completed in August 1997 and data collection took place at a time when the Hong Kong higher education system comprised only nine accredited higher education institutions. This facilitated a high degree of involvement, with seven of the nine institutions actually participating in the scale development procedure. Thus, Hong Kong provided a suitable base for an initial examination of the relevance of quality to institutional performance assessment in higher education.

\section{Method}

The method of scale development was a version of the procedure for constructing Behaviourally Anchored Rating Scales (BARS) (Smith and Kendall, 1963), modified to maximize validity and reliability.

\section{Generation of Examples}

A pilot study involving a small number of senior academics and administrators in Hong Kong higher education gauged the feasibility of generating behavioural examples for BARS in sufficient numbers for the main study to be feasible. Participants were requested to provide examples of good, average and poor organizational behaviour for each of the nine effectiveness dimensions (i.e. Productivity-Efficiency, Quality, Cohesion, Adaptability-Readiness, Information Management-Communication, Growth, Planning-Goal Setting, Human Resources Development, Stability-Control) contained in the Competing Values Model (Quinn and Rohrbaugh, 1981, 1983). Definitions of the nine effectiveness dimensions are contained in Appendix A. These definitions took the descriptions employed in an earlier experiment by Edwards (1986) conducted in a commercial context, and slightly modified them where necessary for a higher educational setting.

The results of the pilot study indicated the feasibility of the main study, and all nine accredited higher education institutions in Hong Kong were then invited to participate in developing the rating scales. Seven institutions accepted the invitation and 32 senior academic and administrative staff in Hong Kong higher education agreed to provide behavioural examples, as in the pilot study. The rationale for inviting only senior staff was the need for participants to occupy positions affording them an overview of institutional performance in order for them to provide examples of behaviour at an institutional level. A total of 592 examples were generated.

\section{Screening of Examples}

The behavioural examples generated were subjected to an initial screening by a panel of four judges at senior institutional management level (i.e. Vice-President, Dean of Business Faculty, Institutional Consultant/Former Associate VicePresident and Head of Management Department) in one of the accredited 
Pounder: The Relevance of Quality to Institutional Performance Assessment

institutions of higher education in Hong Kong. Panel members were advised to screen out any examples which fell into one or more of the following categories:

(a) did not describe behaviour at the institutional level;

(b) expressed sentiments similar to another example perceived to be better constructed;

(c) was a description of an intangible trait rather than a tangible illustration of behaviour;

(d) was worded ambiguously;

(e) described behaviour associated with more than one dimension of effectiveness.

\section{Retranslation}

A randomized list of the examples remaining after screening and a list of the nine effectiveness dimensions, with descriptions, enabled 162 academic and administrative staff in the seven participating institutions to undertake retranslation. This involved the reallocation of a randomized list of examples to relevant dimensions. A 60 percent agreement criterion (i.e. for each example, 60 percent of participants in the retranslation exercise agreeing that the example related to a particular effectiveness dimension) was employed, which permitted the retention of sufficient examples to form scales in all nine effectiveness dimensions contained within the Competing Values Model. Bernardin et al. (1976) have recommended the 60 percent criterion based on a comprehensive examination of the effects of procedural variations on the psychometric properties of BARS scales.

\section{BARS Scaling}

One hundred and thirty academic and administrative staff in the seven participating institutions engaged in the BARS scaling. BARS scaling involved each behavioural example being given a numerical rating by each participating staff member. This rating reflected the participant's perception of the level of performance in a particular effectiveness dimension conveyed by the example in question. Thus, for instance, an example judged by a staff member to represent good performance in a particular effectiveness dimension was given a higher numerical rating than an example considered by that same staff member to represent average or poor performance. In accordance with the BARS procedure, the objective of the scaling was to form rating scales in each of the nine effectiveness dimensions from only those examples with low standard deviations. The BARS procedure requires that those examples then be located as scale anchors according to the mean of their numerical ratings.

Landy and Farr (1980) concluded that the decision rules for scaling are often arbitrary and a survey of literature did reveal variations in the number of scale points used for rating examples and the standard deviation criteria employed for scaling. It was felt that numerical scales exceeding five points would tend to make the rating of behavioural examples too complex, by requiring participants to make excessively fine judgements on levels of performance represented by the examples. Additionally, BARS literature contains a study which developed scales 


\section{Evaluation 6(1)}

capable of valid measurement based on a five-point scaling of anchors and a standard deviation of 1.00 or less (Dickinson and Zellinger, 1980). In developing the BARS for the Hong Kong study, a five-point scale for rating behavioural examples was employed together with a more stringent standard deviation criterion than in the Dickinson and Zellinger study, namely 0.9 or less.

\section{Multidimensional Scaling}

Valid rating requires that rating scales be logically ordered (scalable). The scalability notion, originating with Guttman (1944), requires that scales produce response patterns 'in which endorsement of the item reflecting the extreme position results also in endorsing all items which are less extreme' (Emory and Cooper, 1995: 223). Hence, scales should be ordered in such a way that a rater will not be faced with the dilemma of finding two anchor descriptions on the same scale which seem equally representative of a subject's performance, where one description purports to depict good performance and the other, poor performance. Guttman's analysis has been viewed as a less powerful scale verification technique than contemporary multidimensional analysis, which has become possible as a result of computing developments (Wimberley, 1976).

Multidimensional scaling was employed in the Hong Kong study as a means of validating the ordering of scale anchors. Dissimilarity matrices were prepared for each of the nine effectiveness dimensions to enable pair-wise dissimilarity judgements to be made for all the behavioural examples retained as scale anchors after BARS scaling had been completed. Dissimilarity judgements were made using a five-point numerical scale. The selection of a scale confined to five points was to avoid the need for participants to make excessively fine judgements on degrees of dissimilarity between pairs of behavioural examples. For example, a participant might record a score of one in the appropriate cell of the matrices when a pair of behavioural statements was judged to be very similar, and in the case of a pair of examples judged to be very dissimilar, a score of five might be given. It was recognized that multidimensional scaling requires careful attention to the task on the part of participants and led to the selection of a panel of 25 judges who undertook the exercise. Selection of the judges was on the basis that:

(i) they occupied senior academic or administrative roles in Hong Kong higher education; or

(ii) they had an academic background in organizational analysis and were currently involved in research or teaching in an area related to the study.

It was reasoned that a validly ordered scale would exhibit a pattern of anchor ordering such that anchors representing clear differences in level of performance according to BARS scaling would be judged to be clearly different according to multidimensional scaling. It followed also that anchors representing clearly similar levels of performance should be judged to be clearly similar. Correlation analysis was used to compare the ordering of anchors produced by the BARS and multidimensional scaling methods. Those scales which were judged to possess relatively weak correlations between the rank ordering of examples produced by both scaling methods were eliminated from the study. 


\section{Multitrait-Multirater Analysis}

Valid rating requires that rating scales be unidimensional. This means that descriptive scale anchors should be statements capturing the content of the trait, or in the case of the Hong Kong study, the effectiveness dimension to which they relate and only that trait or effectiveness dimension. This aspect of validity is frequently examined with reference to Campbell and Fiske's (1959) multitraitmultimethod analysis. The multitrait-multirater variant of Campbell and Fiske's analysis was employed in this study, given that the analysis remains current as a tool for gauging the construct validity (unidimensionality) of measurement scales in the human domain (Henderson et al., 1995; Kinicki et al., 1985; Lascu et al., 1995; Schreisheim and Eisenbach, 1995; Spreitzer, 1995; Sullivan, 1996). Furthermore, psychometricians continue to confirm the efficacy of multitraitmultimethod analysis as a thorough approach to validating measurement employing rating scales (Cronbach, 1990; Gregory, 1992; Murphy and Davidshofer, 1991).

Multitrait-multirater analysis requires the production of a matrix which presents all intercorrelations when each of a number of traits (in this study, organizational effectiveness dimensions) is measured by more than one source of ratings. The pattern of intercorrelations indicates the presence, or absence, of construct validity. For the purpose of multitrait-multirater analysis, the scales remaining after the scaling stage of the procedure were formed into an institutional selfrating instrument and completed by 151 academic and administrative staff at various levels of responsibility within the seven participating organizations. Two categories of respondents were identified: Assistant/Associate Professor and Others. The first group was viewed as largely homogeneous in job nature, with a primary emphasis on teaching and research. The second group was considered less homogeneous than the former but commonly those falling into this category tended to perform organizational roles with a high administrative content. This categorization enabled multitrait-multirater analysis to be carried out (Pounder, 1997).

\section{Tests for Reliability}

In addition to the above, the inter-rater reliability and test-retest reliability of rating employing each of the scales was gauged against the Nunnally and Bernstein (1994) recommended reliability coefficient of around 0.70 .

\section{Results and Conclusions}

Multitrait-multirater analysis (Campbell and Fiske, 1959) identified scales capable of construct valid rating in four out of the nine effectiveness dimensions contained in the Competing Values Model (Quinn and Rohrbaugh, 1981, 1983). These scales were in the Information Management-Communications, Planning-Goal Setting, Productivity-Efficiency and Cohesion effectiveness dimensions. For reference, the Cohesion scale is reproduced in Appendix B. Multidimensional scaling confirmed that each of the four scales were scalable. Content validity requirements were generally satisfied because the BARS procedure involves scale development by experts. Additionally, each of the four 


\section{Evaluation 6(1)}

scales met the threshold requirement for reliability. However, in the study, quality was notably absent as an effectiveness dimension found to be valid and reliable for institutional performance rating in Hong Kong higher education. The scale for the Quality Effectiveness Dimension (Appendix C) was eliminated in the scale development procedure on the basis of its lack of unidimensionality, meaning that participants in the procedure perceived this scale to reflect more than one dimension, thus supporting the view that quality defies generally agreed definition (Garvin, 1988).

This article has described one of the few studies that has tested the adequacy of the quality notion as a conceptual base for institutional performance assessment in higher education. The results of the study indicated that the quality notion is viewed as not confined to one dimension of effectiveness, and therefore is unable to provide common ground for a set of behavioural statements designed to reflect quality performance. In brief, an attempt to develop a unidimensional scale for quality led to the generation of a series of behavioural examples which reflected only the specific perceptions of quality held by the providers of the examples. Hence, in highlighting the ambiguity inherent in the quality notion, the Hong Kong study supports the body of opinion in the fields of both commerce and higher education which emphasizes the indeterminate nature of the concept (see the Introduction to this article). A positive outcome of the study was the development of unidimensional (construct valid) and reliable scales in the Information Management-Communications, Planning-Goal Setting, ProductivityEfficiency and Cohesion effectiveness dimensions. On the basis of the Hong Kong experiment, these four concepts have potential for anchoring institutional performance assessment in higher education to a firmer base than that provided by quality alone.

It may be argued that one should not get too preoccupied with defining quality because such a preoccupation can detract from the fact that quality with suffixes such as management, audit, assurance and control has provided the basis for positive and constructive initiatives in higher educational performance. This argument holds true for initiatives that are confined to a particular institution. So, for example, in describing the introduction of a quality assurance system for administrative units in the University of Wales College of Cardiff, Butcher and his colleagues arrived at the following working definition of quality:

... the degree to which agreed standards relating to the priority requirements or needs of users of the College's administrative services are achieved and continuously improved, bearing in mind resourcing levels and other local constraints. (Butcher, 1996: 2)

However, it is likely that the above definition, whilst perfectly adequate for the administrative units of that institution, would not be acceptable as an adequate definition of quality for the academic units of the same institution. Equally, it is possible that administrative units of other institutions may not find the definition suitable for them. In higher education, the quality notion runs into problems when its application goes beyond the particular institution involved. Idiosyncratic definitions of quality lead to idiosyncratic measurement initiatives and results. In 
a situation such as that which is currently prevailing in higher education, where institutional performance evaluation is not anchored to a generally agreed definition of quality, at best international comparisons of institutional performance, and at worst inter-institutional comparisons of performance within a particular country, are problematic. The challenge for higher education is to locate concepts that, in complementing quality and adding precision to institutional performance assessment in higher education, facilitate international and inter-institutional comparisons of performance. The study described in this article has indicated a method which has the potential for identifying such concepts.

\section{Appendix A: Competing Values Dimensions - Definitions Employed in the Study}

- Productivity-Efficiency: This aspect of an institution's performance has to do with behaviour that reflects the extent to which it is concerned with the quantity or volume of what it produces and the cost of operation.

- Quality: This aspect of an institution's performance has to do with behaviour that reflects the extent to which it is concerned with the quality of what it produces.

- Cohesion: This aspect of an institution's performance has to do with behaviour that reflects the extent to which it is concerned with staff morale, interpersonal relationships, teamwork and sense of belonging.

- Adaptability-Readiness: This aspect of an institution's performance has to do with behaviour that reflects the extent of its ability to readily alter or adapt its structure, programmes, courses etc., in response to changing demands. In other words, the extent of the organization's readiness to adapt to change.

- Information Management-Communication: This aspect of an institution's performance has to do with behaviour that reflects the extent of its ability to distribute timely and accurate information needed by its members to do their jobs.

- Growth: This aspect of an institution's performance has to do with behaviour that reflects the extent of its ability to secure external support, acquire resources and increase its capabilities.

- Planning-Goal Setting: This aspect of an institution's performance has to do with behaviour that reflects the extent of its ability to set goals and objectives and systematically plan for the future.

- Human Resource Development: This aspect of an institution's performance has to do with behaviour that reflects the extent to which it is responsive to the individual needs of its staff. It also has to do with the extent to which the institution facilitates participation in decision making. Additionally, this aspect is concerned with behaviour relating to the hiring, training and development of staff.

- Stability-Control: This aspect of an institution's performance has to do with behaviour that reflects the extent of its ability to control the flow of work, to direct the behaviour of its members and to maintain the organization's continuity. 


\section{Appendix B: Performance Dimension - Cohesion}

Definition: This aspect of an institution's performance has to do with behaviour which reflects the extent to which it is concerned with staff morale, interpersonal relationships, teamwork and sense of belonging.

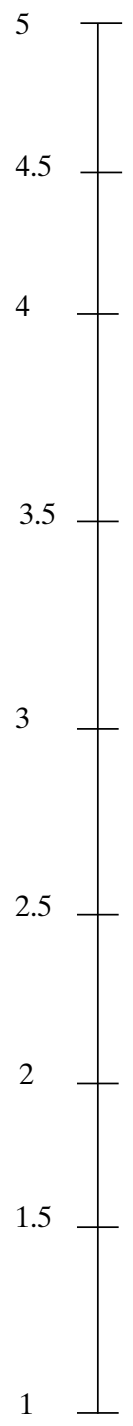

Typically, in this institution, one would expect senior management to have taken positive steps to create a climate in which employees at all levels are made to feel valuable members of the institution.

Typically, in this institution, one would expect staff to regularly refer to their sense of commitment to the institution.

Typically, in this institution, one would expect mechanisms to exist for staff to share problems and to work together.

Typically, in this institution, one would expect the senior management team to visit academic and administrative units regularly and talk freely and informally with members of staff.

Typically, in this institution, one would expect there to have been an effort either to foster allegiance to parts of the organization in the face of growth or to limit expansion so as to maintain a sense of belonging.

Typically, in this institution, one would expect staff to demonstrate greater allegiance to the parent Faculty or Department than to the institution as a whole.

Typically, in this institution, one would expect senior management to express a commitment to the maintenance of staff morale but not to do sufficient to ensure that people at all levels have a sense of belonging.

Typically, in this institution, one would expect there to be frequent conflicts between the centre and sub-units, between academic and administrative units, between one academic or administrative unit and another, and between individuals.

Typically, in this institution, one would expect feelings of loyalty and sense of belonging to be undermined by an approach to human relations issues (e.g. to contract renewal) which generates insecurity.

Typically, in this institution, one would expect there to be a general lack of informal contact amongst staff members demonstrated by inadequate staff common room life and too many closed office doors.

This space below is provided for raters to write down their own example (optional - see rater's instructions): 


\section{Appendix C: Performance Dimension - Quality}

Definition: This aspect of an institution's performance has to do with behaviour that reflects the extent to which it is concerned with the quality of what it produces.

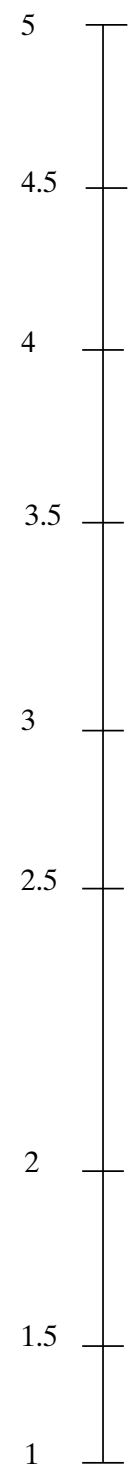

Typically, in this institution, one would expect a variety of sources (e.g. formal peer review, student responses, relevant statistics, views of staff, opinions of external examiners and employers) to be utilized for evaluating and monitoring quality.

Typically, in this institution, one would expect mechanisms to be in place (e.g. procedures for assessment of 'value added', questionnaires to employers) to monitor the quality of its graduates.

Typically, in this institution, one would expect there to be a general acceptance that the major constraint on increasing student numbers must be the maintenance of academic standards.

Typically, in this institution, one would expect a relatively narrow view of quality to be taken, with the result that one or two limited measures (e.g. students' grade point averages, graduate employment) are considered to be sufficient indicators of the institution's performance in this area.

Typically, in this institution, one would expect the external examiner system to be the main mechanism for monitoring quality.

Typically, in this institution, one would expect quality to be extolled and recognized where it exists but no positive action on the part of senior management to be taken to foster a quality culture.

Typically, in this institution, one would expect quality to be evaluated sporadically and unsystematically, usually in response to a problem and not as a matter of policy.

Typically, in this institution, one would expect there to be no system for monitoring the quality of continuous assessment.

Typically, in this institution, one would expect there to be little attention paid to generating commitment to quality.

This space below is provided for raters to write down their own example (optional - see rater's instructions): 


\section{References}

Bauer, M. (1992) 'Evaluation Criteria and Evaluation Systems', in A. Craft (ed.) Quality Assurance in Higher Education: Proceedings of an International Conference, Hong Kong, 1991. London: Falmer.

Bernardin, H. J., M. B. LaShells, P. C. Smith and K. M. Alvares (1976) 'Behavioral Expectation Scales: Effects of Developmental Procedures and Formats', Journal of Applied Psychology 61: 75-9.

Broh, R. A. (1982) Managing Quality for Higher Profits. New York: McGraw-Hill.

Butcher, R. (1996) 'The Introduction of an Administrative Quality Assurance System Some Cultural Questions', paper presented at the International Conference on Quality Assurance and Evaluation in Higher Education, Beijing: Chinese Society of Higher Education Evaluation, People's Republic of China/Hong Kong Council for Academic Accreditation.

Cameron, K. S. and S. J. Freeman (1989) 'Cultural Congruence, Strength and Type: Relationship to Effectiveness', working paper, Ann Arbor: University of Michigan, School of Business.

Campbell, J. P. and D. W. Fiske (1959) 'Convergent and Discriminant Validation by the Multitrait-Multimethod Matrix', Psychological Bulletin 36: 81-105.

Cronbach, L. J. (1990) Essentials of Psychological Testing. New York: HarperCollins.

Dickinson, T. L. and P. M. Zellinger (1980) 'A Comparison of the Behaviorally Anchored Rating and Mixed Standard Scale Formats', Journal of Applied Psychology 65: 147-541.

DiPadova, L. N. and S. R. Faerman (1993) 'Using the Competing Values Framework to Facilitate Managerial Understanding Across Levels of Organisational Hierarchy', Human Resources Management 32(1): 143-74.

Dunk, A. and A. F. Lysons (1997) 'An Analysis of Departmental Effectiveness, Participative Budgetary Control Processes and Environmental Dimensionality within the Competing Values Framework: A Public Sector Study', Financial Accountability and Management 13(1): 1-15.

Edwards, R. L. (1986) 'Using Multidimensional Scaling to Test the Validity of Behaviourally Anchored Rating Scales: An Organisational Example Involving the Competing Values Framework', PhD dissertation, State University of New York at Albany.

Emory, C. W. and D. R. Cooper (1995) Business Research Methods. Boston, MA: Irwin.

Garvin, D. A. (1988) Managing Quality: The Strategic and Competitive Advantage. New York: Free Press.

Giek, D. G. and P. L. Lees (1993) 'On Massive Change: Using the Competing Values Framework to Organise the Educational Efforts of the Human Resources Function in New York State Government', Human Resources Management 32(1): 9-28.

Gilmore, H. L. (1974) 'Product Conformance Cost', Quality Progress June: 16.

Goedegebuure, L. C. J., P. A. M. Maassen and D. F. Westerhiejden (1990) 'Quality Assessment in Higher Education', in L. C. J. Goedegebuure, P. A. M. Maassen and D. F. Westerheijden (eds) Quality Assessment in British and Dutch Higher Education. Utrecht: Lemma.

Gregory, R. J. (1992) Psychological Testing: History, Principles and Applications. Boston, MA: Allyn and Bacon.

Guttman, L. (1944) 'A Basis for Scaling Qualitative Data', American Sociological Review 9: $139-50$.

Hart, S. L. and R. E. Quinn (1993) 'Roles Executives Play: CEO's, Behavioural Complexity, and Firm Performance', Human Relations 46: 543-74. 


\section{Pounder: The Relevance of Quality to Institutional Performance Assessment}

Henderson, F., N. Anderson and S. Rick (1995) 'Future Competency Profiling: Validating and Redesigning the ICL Graduate Assessment Centre', Personnel Review 24(3): 19-31.

Hooijberg, R. and F. Petrock (1993) 'On Cultural Change: Using the Competing Values Framework to Help Leaders Execute a Transformational Strategy', Human Resources Management 32(1): 29-50.

Kinicki, A., B. Bannister, P. Hom and A. DeNisi (1985) 'Behaviorally Anchored Rating Scales vs Summated Rating Scales: Psychometric Properties and Susceptibility to Rating Bias', Educational and Psychological Measurement 45: 535-49.

Kuehn, A. A. and R. L. Day (1962) 'Strategy of Product Quality', Harvard Business Review 40(6): 101.

Landy, F. J. and J. L. Farr (1980) 'Performance Rating', Psychological Bulletin 87: 72-107.

Lascu, D. N., N. Ashworth, T. Giese and M. Omar (1995) 'The User Information Satisfaction Scale: International Applications and Implications for Management and Marketing', Multinational Business Review 3(2): 107-15.

Liaison Committee of Rectors' Conferences (1993) Quality Assessment in European University and Higher Education. Brussels.

Lucier, P. (1992) 'Performance Indicators in Higher Education: Lowering the Tension of the Debate', Higher Education Management 4: 204-14.

McGraw, R. B. (1993) 'Union-Management Interface: Using the Competing Values Framework as a Diagnostic Tool to Bring Increased Involvement at the Plant Level', Human Resources Management 32(1): 51-73.

Murphy, K. R. and C. O. Davidshofer (1991) Psychological Testing: Principles and Applications. Englewood Cliffs, NJ: Prentice-Hall.

Nunnally, J. C. and I. H. Bernstein (1994) Psychometric Theory. New York: McGraw-Hill.

Pirsig, R. M. (1974) Zen and the Art of Motorcycle Maintenance. New York: Bantam Books.

Pounder, J. S. (1997) 'Measuring the Performance of Institutions of Higher Education in Hong Kong: An Organisational Effectiveness Approach', PhD dissertation, Brunel University/Henley Management College.

Quinn, R. E. and J. Rohrbaugh (1981) 'A Competing Values Approach to Organisational Effectiveness', Public Productivity Review 5(2): 122-40.

Quinn, R. E. and J. Rohrbaugh (1983) 'A Spatial Model of Effectiveness Criteria: Towards a Competing Values Approach to Organisational Analysis', Management Science 29(3): 363-77.

Quinn, R. E. and G. M. Spreitzer (1991) 'The Psychometrics of the Competing Values Culture Instrument and an Analysis of the Impact of Organizational Culture on Quality of Life', in R. W. Woodman and W. A. Pasmore (eds) Research in Organizational Change and Development. Greenwich, CT: JAI Press.

Quinn, R. E., H. W. Hildebrandt, P. S. Rogers and M. P. Thompson (1991) 'A Competing Values Framework for Analysing Presentational Communication in Management Contexts', Journal of Business Communication 28(3): 213-32.

Reeves, C. A. and D. A. Bednar (1994) 'Defining Quality: Alternatives and Implications', Academy of Management Review 19(3): 419-45.

Rogers, P. S. and H. W. Hildebrandt (1993) 'Competing Values Instruments for Analysing Written and Spoken Management Messages', Human Resources Management 32(1): 121-42.

Schreisheim, C. A. and R. J. Eisenbach (1995) 'An Exploratory and Confirmatory FactorAnalytic Investigation of Item Wording Effects on the Obtained Factor Structures of Survey Questionnaire Measures', Journal of Management 21(6): 1177-93.

Segers, M. S. R., W. H. F. W. Wijnen and F. J. R. C. Dochy (1990) 'Performance Indicators: 


\section{Evaluation 6(1)}

A New Management Technology for Higher Education?', in F. J. R. C. Dochy, M. S. R. Segers and W. H. F. W. Wijnen (eds) Management Information and Performance Indicators in Higher Education: An International Issue. Assen/Maastricht: Van Gorcum.

Sendelbach, N. B. (1993) 'The Competing Values Framework for Management Training and Development: A Tool for Understanding Complex Issues and Tasks', Human Resources Management 32(1): 75-99.

Sizer, J., A. Spee and R. Bormans (1992) 'The Role of Performance Indicators in Higher Education', Higher Education 24: 133-5.

Smith, P. C. and L. M. Kendall (1963) 'Retranslation of Expectations: An Approach to the Construction of Unambiguous Anchors for Rating Scales', Journal of Applied Psychology 47: 149-55.

Spreitzer, G. M. (1995) 'Psychological Empowerment in the Workplace: Dimensions, Measurement, and Validation', Academy of Management Journal 38(5): 1442-65.

Staropoli, A. (1992) 'The French Comite National d'Evaluation', in A. Craft (ed.) Quality Assurance in Higher Education: Proceedings of an International Conference, Hong Kong, 1991. London: Falmer.

Stevens, B. (1996) 'Using the Competing Values Framework to Assess Corporate Ethical Codes', Journal of Business Communication 33(1): 71-84.

Sullivan, D. (1996) 'Measuring the Internationalization of a Firm: A Reply', Journal of International Business Studies 27(1): 179-92.

Vroeijenstijn, T. (1992) 'External Quality Assessment, Servant of Two Masters? The Netherlands University Perspective', in A. Craft (ed.) Quality Assurance in Higher Education: Proceedings of an International Conference, Hong Kong, 1991. London: Falmer.

Waller, M. J., G. P. Huber and W. H. Glick (1995) 'Functional Background as a Determinant of Executives' Selective Perception', Academy of Management Journal 38(4): 943-74.

Williams, G. (1990) 'Quality and Resources Allocation', in C. P. J. Loder (ed.) Quality Assurance and Accountability in Higher Education. London: Kogan Page.

Wimberley, R. C. (1976) 'Alam and Alas: Questioning Error Assignment in Unidimensional Guttman Scaling', Educational and Psychological Measurement 36: 361-7.

Yeung, A. K. O., J. W. Brocklebank and D. O. Ulrich (1991) 'Organisational Culture and Human Resources Practices: An Empirical Assessment', working paper, Ann Arbor, University of Michigan, School of Business.

Zammuto, R. F. and J. Y. Krakower (1989) 'Quantitative and Qualitative Studies of Organisational Culture', Academy of Management Paper.

Zammuto, R. F. and E. J. O'Connor (1992) 'Gaining Advanced Manufacturing Technologies' Benefits: The Roles of Organisation Design and Culture', Academy of Management Review 17(4): 701-28.

\footnotetext{
JAMES S. POUNDER is Associate Professor in Management at Lingnan

College, Tuen Mun, Hong Kong. His current research is in the area of organizational performance measurement in higher education. Please address correspondence to Lingnan College, Tuen Mun, Hong Kong. [email: pounder@In.edu.hk]
} 